\title{
Design of an advanced hot box-cold box with full climatic control to test heat, air and moisture transport through real scale building elements
}

\author{
David Daems ${ }^{1, *}$, Antoine Tilmans ${ }^{1}$, Timo De Mets ${ }^{1}$, and Xavier Loncour ${ }^{1}$ \\ ${ }^{1}$ Belgian Building Research Institute, Laboratoire Hygrothermie, Rue du Lombard 42, 1000 Bruxelles, Belgium
}

\begin{abstract}
A versatile hot box-cold box test facility called HAMSTER enables to evaluate the energy and hygrothermal performance of up to $3 \mathrm{~m}$ high, $3 \mathrm{~m}$ wide and $3.6 \mathrm{~m}$ deep building test elements including walls, flat or pitched roofs and floors. This equipment allows for the precise measurement of thermal performance according to standards. It also allows for the study of heat, air and moisture phenomena taking place in building elements under realistic climatic conditions including the effects of rain, sun or wind. Interior and exterior climates are simulated in, respectively, the hot and cold chambers by controlling the temperature, relative humidity, pressure difference between both chambers, infra-red or solar simulator heating and water spraying in the cold chamber. Critical issues that have been solved during the design and construction phases in order to reach both the required performances and the versatility of the test facility are discussed.
\end{abstract}

\section{Introduction}

As the energy consumption of buildings must decrease drastically, innovative materials, building components and construction methods are regularly appearing. There is an increasing need for reliable information on the hygrothermal performance of these elements. While calculation methods are most of the time used, either they are limited to simple building elements, well-known transport phenomena and materials, or they involve complex simulation models that require many, sometimes insufficiently known, input parameters. To address these limitations, an advanced hot box-cold box test facility called HAMSTER has been built. This equipment allows for the evaluation of the purely thermal or hygrothermal performance of building elements up to $3 \mathrm{~m}$ high, $3 \mathrm{~m}$ wide and $3.6 \mathrm{~m}$ deep under realistic climatic conditions involving combined heat, air and moisture transfer. In order to reach the required performances and the expected measurements accuracy on the one hand and allowing for the versatility of the equipment on the other hand, a number of critical issues have been solved during the design and construction phases. Section 2 provides a general description of the facility. The various tests and configurations of the equipment are described in section 3. Critical issues such as the thermopile and electric guard of the hot box walls or the specific design of test frames are discussed in section 4. Conclusions are then given.

\section{General description of the HAMSTER test facility}

The HAMSTER test facility is designed to study the thermal or hygrothermal performance of a variety of real scale building elements such as walls, flat or pitched roofs, floors or construction details. It consists of a hot box and a cold box which respectively allow for the simulation of internal and external climates. The building element to be tested - up to $3 \mathrm{~m}$ high, $3 \mathrm{~m}$ wide and $3.6 \mathrm{~m}$ deep - is inserted into a frame and placed between the boxes. A schematic view with several types of building elements is displayed in Figure 1.
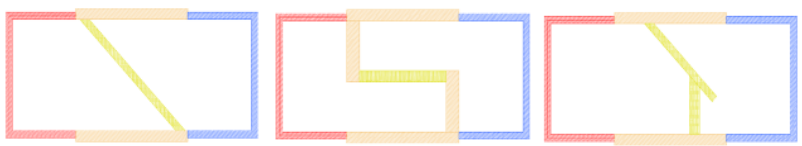

Fig. 1. Vertical sections of the HAMSTER test facility with various building elements to be tested: pitched roof (left), flat roof (centre) and construction detail (right).

A stand-alone metering box can be placed inside the hot box to carry out certain thermal tests. The cold box is equipped with a mobile water spraying system in order to simulate raining conditions and with an infra-red radiation heating. The back wall of the cold box is also equipped with a glazed section which allows for radiations emitted by an external solar simulator to irradiate the test wall while monitoring the cold side atmospheric parameters.

\footnotetext{
* Corresponding author: david.daems@ bbri.be
} 


\section{Types of tests and configurations of the equipment}

The HAMSTER test facility allows for the realisation of a wide variety of tests which can be grouped into three categories described in the next three subsections.

\subsection{Purely thermal performance measurements}

These tests amount to determining the heat transfer properties of building walls such as the thermal transmittance (U-value). They are associated to standards (in particular EN ISO 8990:1996, EN 19464:2000, EN ISO 12567-1:2010 and EN 1934:1998) which allow for the definition of very precise methodology and equipment to be employed in testing.

The aim is to measure precisely the heat flow that passes through the portion of the test wall, read inside the metering box (value $\phi_{1}$ ), based on the heat flows injected into the metering box (via electrical resistance and/or fans, value $\phi_{\mathrm{h}}$ and $\phi_{\mathrm{f}}$ respectively) to maintain the system in steady-state operations (for example, $20^{\circ} \mathrm{C}$ in the metering box and $0^{\circ} \mathrm{C}$ in the cold box). In order to adjust precisely the heat exchange coefficients between the air and the wall, baffles equipped with fans are placed in each box in front of the test wall. To achieve the required precision, efforts are made to minimise the heat flow that passes through the metering box walls (value $\phi_{3}$ ) and through the side section of the wall section measured (value $\phi_{2}$ ) so that the flow measurement of heat injected into the metering chamber is as close as possible to the heat passing through the test wall $\left(\phi_{\mathrm{h}}+\phi_{\mathrm{f}}->\phi_{1}\right)$.

The HAMSTER equipment can be operated in 2 modes which differ in the method for cancelling the heat flow through the walls of the metering box. In "wallguarded hot box" mode, the metering box is the hot box and an active electric guard integrated inside the walls of this box is used to cancel the heat flow crossing over them. This mode is illustrated in Figure 2.

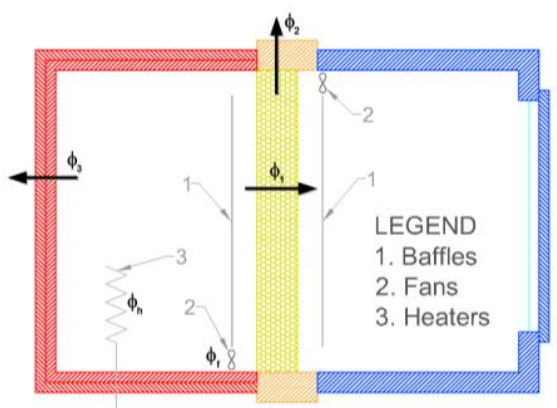

Fig. 2. Wall-Guarded Hot Box mode for purely thermal measurements.

In "guarded hotbox" mode, a stand-alone metering box is used and the hot box serves as the guard box. The heat flow through the walls of the stand-alone metering box is cancelled by controlling appropriately the temperature of the air located between the hot box and the stand-alone metering box. This mode, which gives rise to a higher precision, is illustrated in Figure 3.

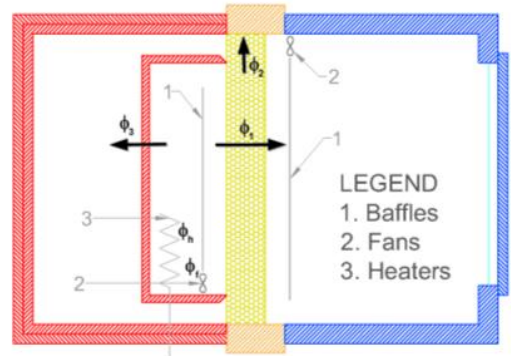

Fig. 3. Guarded Hot Box mode for purely thermal measurements.

\subsection{Tests of heat, air and moisture transports}

The tests allowing for a combined study of Heat, Air and Moisture transfer phenomena (HAM) within building elements will be referred to as "HAM tests". The HAMSTER test facility allows one to simulate realistic internal and external climates in, respectively, the cold and hot boxes:

- The temperature and relative air humidity in each box are regulated.

- A mobile spray boom that travels vertically parallel to the test element in the cold box can simulate precipitation.

- A pressure difference can be established between the cold box and hot box atmospheres in order to generate an infiltration flow through test elements that are not airtight.

- Two operating modes are available. In the first mode, a surface heating of the test element is obtained by an infra-red source which is placed in the cold box. This mode is illustrated in Figure 4.

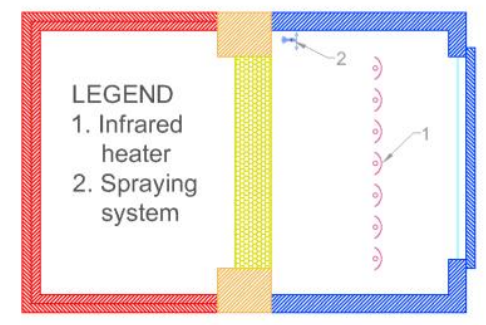

Fig. 4. HAM tests with infra-red heating.

It is also available for non-vertical walls such as flat roofs or pitched roofs as illustrated in Figure 5.

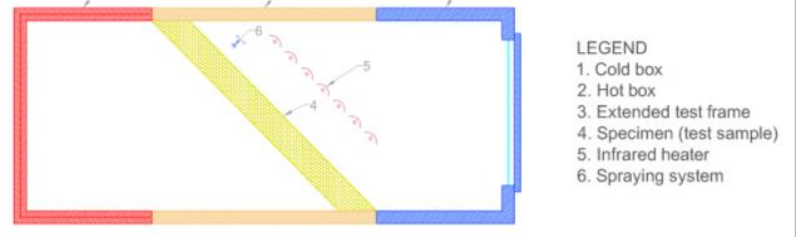

Fig. 5. HAM tests of non-vertical elements with infra-red heating. 
In the second mode, a solar simulator is placed outside the cold box with the backside glazing protection doors of the cold box in the open position. This mode is illustrated in Figure 6.

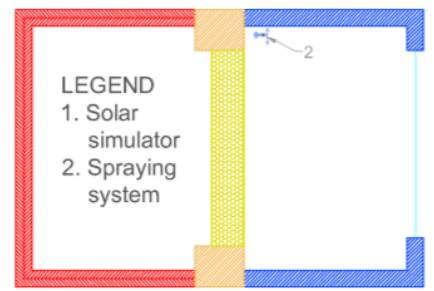

Fig. 6. HAM tests with solar simulator.

\subsection{Air permeability tests}

Air permeability tests can be conducted with a metal box that is fixed to the different test frames or the hot and cold boxes. This is illustrated in Figure 7.
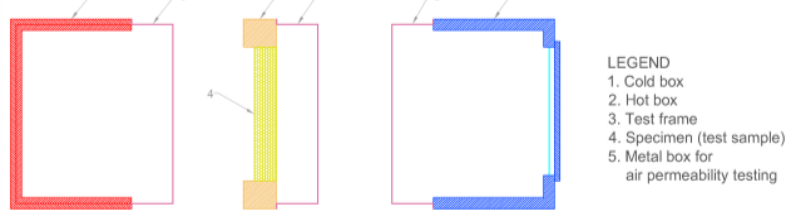

Fig. 7. Air permeability tests of walls

\section{Critical design issues}

A general view of the HAMSTER equipment is displayed in Figure 8 with the hot box in front, the extended test frame (as illustrated in Figure 1) in the middle and the cold box at the back.

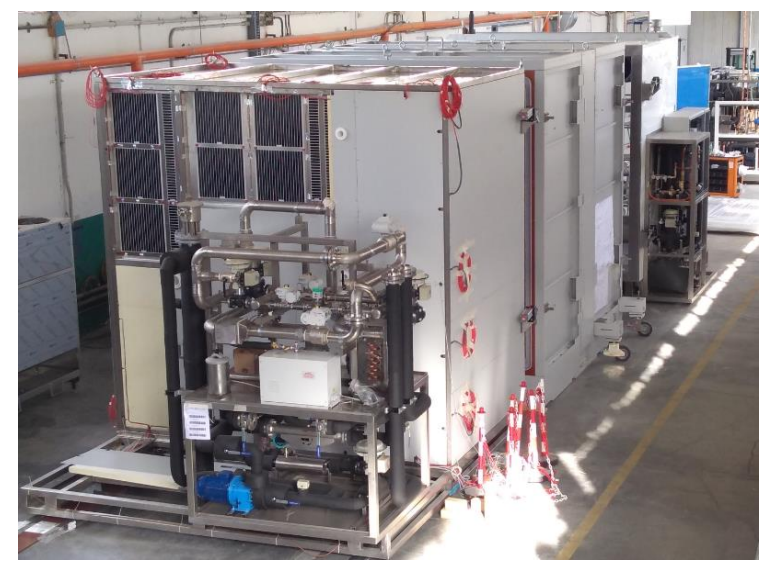

Fig. 8. General view of the HAMSTER equipment: Hot box (front), extended test frame (middle), cold box (back).

For purely thermal measurements according to standards, the HAMSTER test facility can be configured in the "wall-guarded hot box" mode described in section 3.1 and illustrated in Figure 2. In this configuration, the metering box serves as the hot box and an active electric guard is integrated inside the walls of the box in order to cancel the heat flow crossing over them by adjusting the temperature of the external wall of the box with the help of heating pads. The temperature difference within the walls of the hot box is measured with a thermopile which is installed across the hot box walls. Several existing equipments incorporate this operating principle [1-3].

The arrays of thermocouples constituting the thermopiles of the hot box are visible on Figure 9 (exterior side, during construction) and Figure 10 (interior side, with a cover panel removed for illustration). There are 6 thermopiles for the hot box: one for each side and one for the door (whose entrance is visible on the right side of Figure 10).

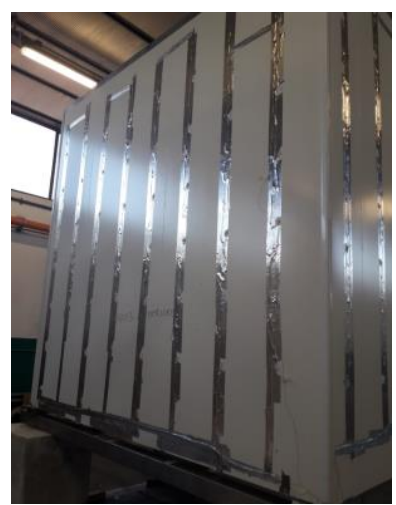

Fig. 9. Thermocouple array constituting the exterior side of the thermopile of the hot box (during construction).

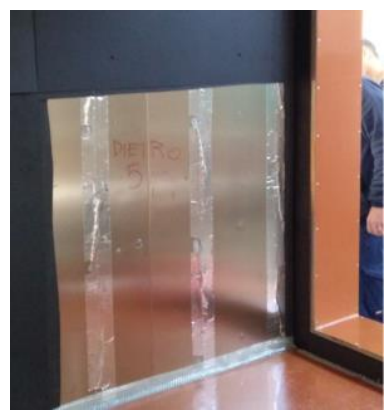

Fig. 10. Thermocouple array constituting the interior side of the thermopile of the hot box (interior black panel removed for illustration).

The surface electric heaters constituting the active electric guard of the hot box are visible on the upper left part of Figure 8 (external panels removed for illustration) and on Figure 11 during the construction phase. 


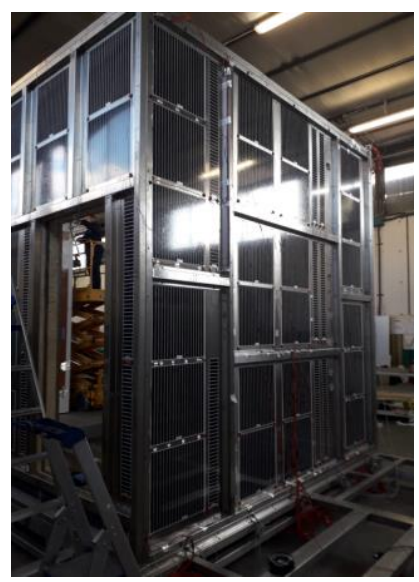

Fig. 11. Surface electric heaters of the active guard of the hot box (construction phase).

In order to minimize the lateral heat losses through the test frame (value $\phi_{2}$ of Figures 2 and 3), numerical simulations were performed to determine the appropriate level of insulation of the frame while also ensuring rigidity and the possibility to fasten building elements. This is illustrated in Figure 12, both during construction (when wooden beams for rigidity and fastening were still visible) and after completion of the construction.

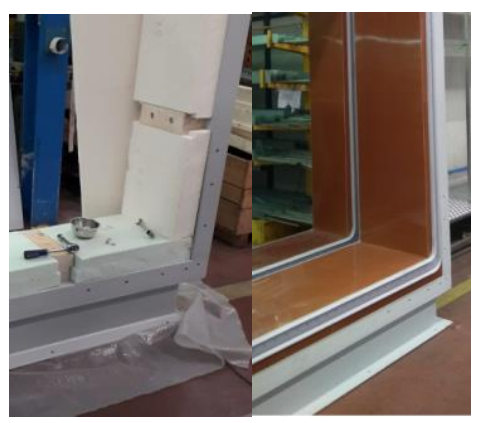

Fig. 12. Test frame (construction phase on the left side, complete on the right side)

These elements make it possible to significantly reduce the heat flow passing through the walls of the hot box by adjusting the temperature of the electrical guard in such a way as to cancel the average temperature difference within the walls of the hot box. As a result, purely thermal performance measurements can be achieved with the level of accuracy required by the pertaining standards.

Given the size, the level of accuracy and the versatility of the equipment, a number of other critical issues were addressed (infra-red heating, spraying system, control system, etc). They cannot all be described here.

\section{Conclusions}

The HAMSTER test facility promotes the sustainability of constructions by targeting the improvement of the energy performance of buildings and the implementation of robust solutions over time. At present, scientific knowledge on the sustainability of construction systems is insufficient. The HAMSTER equipment will contribute to improving this knowledge to advance sustainable development and encourage innovation in the construction sector. The versatility of the test equipment allows to study concretely and quantitatively with a high level of accuracy the questions that arise in the context of the renovation of existing buildings, including heritage, and the construction of new buildings with high energy performance.

Funding by the European Regional Development Fund and Innoviris (Brussels Region, Belgium) are acknowledged.

\section{References}

1. E. Maldonado, "Upgrading PASSYS test cells with a pseudo-adiabatic shell", Report from COMPASS research funded by EU, 1995;

2. Case Williams, "A new approach to the measurement of thermal transmittance; the NPL Wall-Guarded Hot Box", National Physical Laboratory, 1992;

3. Case Williams, G. Ballard, "Validation of the NPL rotatable wall guarded hot box with horizontal heat flow", National Physical Laboratory, 2003 